\title{
Influence of field of view size on image quality: ultra-high-resolution CT vs. conventional high-resolution CT
}

\author{
Tomo Miyata $^{1}$ - Masahiro Yanagawa ${ }^{1} \cdot$ Akinori Hata $^{2} \cdot$ Osamu Honda $^{1} \cdot$ Yuriko Yoshida $^{1} \cdot$ Noriko Kikuchi $^{1}$. \\ Mitsuko Tsubamoto ${ }^{2} \cdot$ Shinsuke Tsukagoshi $^{3} \cdot$ Ayumi Uranishi $^{3} \cdot$ Noriyuki Tomiyama $^{1}$
}

Received: 31 December 2019 / Accepted: 3 February 2020 / Published online: 18 February 2020

(C) The Author(s) 2020

\begin{abstract}
Objectives This study was conducted in order to compare the effect of field of view (FOV) size on image quality between ultrahigh-resolution CT (U-HRCT) and conventional high-resolution CT (HRCT).

Methods Eleven cadaveric lungs were scanned with U-HRCT and conventional HRCT and reconstructed with five FOVs (40, $80,160,240$, and $320 \mathrm{~mm}$ ). Three radiologists evaluated and scored the images. Three image evaluations were performed, comparing the image quality with the five FOVs with respect to the 160-mm FOV. The first evaluation was performed on conventional HRCT images, and the second evaluation on U-HRCT images. Images were scored on normal structure, abnormal findings, and overall image quality. The third evaluation was a comparison of the images obtained with conventional HRCT and U-HRCT, with scoring performed on overall image quality. Quantitative evaluation of noise was performed by setting ROIs.

Results In conventional HRCT, image quality was improved when the FOV was reduced to $160 \mathrm{~mm}$. In U-HRCT, image quality, except for noise, improved when the FOV was reduced to $80 \mathrm{~mm}$. In the third evaluation, overall image quality was improved in U-HRCT over conventional HRCT at all FOVs. Noise of U-HRCT increased with respect to conventional HRCT when the FOV was reduced from 160 to $40 \mathrm{~mm}$. However, at 240- and 320-mm FOVs, the noise of U-HRCT and conventional HRCT showed no differences.

Conclusions In conventional HRCT, image quality did not improve when the FOV was reduced below $160 \mathrm{~mm}$. However, in UHRCT, image quality improved even when the FOV was reduced to $80 \mathrm{~mm}$.

Key Points

- Reducing the size of the field of view to $160 \mathrm{~mm}$ improves diagnostic imaging quality in high-resolution CT.

- In ultra-high-resolution CT, improvements in image quality can be obtained by reducing the size of the field of view to $80 \mathrm{~mm}$.

- Ultra-high-resolution CT produces images of higher quality compared with conventional HRCT irrespective of the size of the field of view.
\end{abstract}

Keywords Image enhancement $\cdot$ Diagnostic imaging $\cdot$ Lung diseases

Abbreviations

Conventional

HRCT

CT
Conventional high-resolution computed

tomography

Computed tomography
CT dose index

FOV

HRCT

ROI
Computed tomography dose index

Field of view

High-resolution CT

Region of interest

Electronic supplementary material The online version of this article (https://doi.org/10.1007/s00330-020-06704-0) contains supplementary material, which is available to authorized users.

Tomo Miyata

t-miyata@radiol.med.osaka-u.ac.jp

1 Department of Radiology, Osaka University Graduate School of Medicine, 2-2 Yamadaoka, Suita City, Osaka 565-0871, Japan
2 Department of Future Diagnostic Radiology, Osaka University Graduate School of Medicine, 2-2 Yamadaoka, Suita City, Osaka 565-0871, Japan

3 Department of CT Systems, Canon Medical Systems Corp., Otawara, Tochigi, Japan 
SD Standard deviation

SNR Signal-to-noise ratio

U-HRCT Ultra-high-resolution computed tomography

\section{Introduction}

Smaller fields of views (FOVs) in CT are generally associated with higher spatial resolution and clearer images. As the image quality becomes clearer, the ability to find lung nodules and the diagnostic ability to distinguish between benign and malignant nodules are improved [1, 2]. However, noise is increased and image quality deteriorated by reducing the $\operatorname{FOV}[3,4]$. Recently, ultra-high-resolution CT (U-HRCT) has been developed, characterized by a smaller focal size of the detector elements and the X-ray tube compared with conventional highresolution CT (conventional HRCT), allowing clearer CT images [5]. U-HRCT with $1024 \times 1024$ matrix size has been reported to improve image quality and diagnostic potential [6], and that a U-HRCT matrix size of $2048 \times 2048$ leads to larger improvement than sizes $512 \times 512$ or $1024 \times 1024$ [7]. However, no studies investigated the influence of FOV size on image quality in U-HRCT. The spatial resolution of conventional HRCT has been reported to range from 0.23 to $0.35 \mathrm{~mm}$, and that of U-HRCT to be $0.14 \mathrm{~mm}[5,7-9]$. One pixel of matrix size $(512 \times 512)$ corresponds to $0.156 \mathrm{~mm}$ $(0.078 \mathrm{~mm})$ when the FOV size is $80 \mathrm{~mm}(40 \mathrm{~mm})$. If the pixel size at a given FOV is smaller than the spatial resolution of conventional HRCT, the image quality cannot improve even if the FOV is further decreased. Therefore, we hypothesized that even at FOV sizes for which the image quality is not further improved in conventional HRCT, an improvement could still be seen in U-HRCT. The purpose of our study was to compare the effect of FOV size on image quality in U-HRCT and conventional HRCT, so as to test such hypothesis.

\section{Materials and methods}

\section{Cadaveric lungs}

The study was approved by our Institutional Review Board and did not require informed consent due to its retrospective design. Eleven cadaveric lungs from patients with a history of pulmonary disease were used. The lungs were inflated and fixed using Heitzman's method [10], namely distended through the main bronchus with fixative fluid containing polyethylene glycol 400, 95\% ethyl alcohol, 40\% formalin, and water in the proportion of 10:5:2:3. The specimens were immersed in the fixative fluid for 2 days and then air-dried.

These samples were pathologically characterized as tuberculosis $(n=2)$, usual interstitial pneumonia $(n=2)$, diffuse pan bronchiolitis, metastatic disease, cardiogenic edema, diffuse alveolar hemorrhage, diffuse alveolar damage, emphysema, and lymphangitic carcinomatosis $(n=1$ each).

\section{U-HRCT and conventional HRCT scanners}

U-HRCT (Aquilion Precision ${ }^{\mathrm{TM}}$; Canon Medical Systems) and conventional HRCT (Aquilion ONETM; Canon Medical Systems) were used as CT scanners. In U-HRCT, the detector element size is $0.25 \times 0.25 \mathrm{~mm}$, with 160 rows and 1792 channels, and the focus size of the X-ray tube is $0.4 \times$ $0.5 \mathrm{~mm}$. On the other hand, in conventional HRCT, the detector element size is $0.5 \times 0.5 \mathrm{~mm}$, with 320 rows and 896 channels, and the focus size of the X-ray tube is $0.8 \times 0.9 \mathrm{~mm}$. The detector size of the U-HRCT scanner is thus half the size of the conventional CT detector elements in both the $x-y$ plane and the $z$-axis direction, and the focus size of the $\mathrm{X}$-ray tube in $\mathrm{U}$ HRCT is approximately $1 / 3$ that of conventional HRCT.

\section{Image acquisition}

The lungs were scanned with U-HRCT and conventional HRCT. U-HRCT images were obtained with a gantry rotation of $1.5 \mathrm{~s}$, pitch factor $0.81,120 \mathrm{kV}, 200 \mathrm{~mA}$, and CT dose index 23.2 mGy. Conventional HRCT images were obtained with a gantry rotation of $1.5 \mathrm{~s}$, pitch factor $0.81,120 \mathrm{kV}, 200 \mathrm{~mA}$, and CT dose index $23.9 \mathrm{mGy}$. Each lung was scanned at three different position levels. CT images with slice thickness $0.5 \mathrm{~mm}$ were reconstructed using the following FOV sizes: $40,80,160,240$, and $320 \mathrm{~mm}$. The images were reconstructed at a $512 \times 512$ matrix size. FC81 was used as the reconstruction kernel (Figs. 1 and 2). In total, we obtained 330 images (66 per each FOV size).

\section{Evaluation of image quality}

To clarify the influence of FOV in conventional HRCT and UHRCT, three image quality evaluations were performed. In the first evaluation, conventional HRCT image quality was compared among the FOV values. In the second evaluation, the same comparison was performed using the U-HRCT images. In the third evaluation, the image quality of conventional HRCT was compared with that of U-HRCT. Image quality was evaluated using an 8.3-megapixel, 32-in. color liquid crystal display monitor.

\section{Evaluation of conventional HRCT image quality}

In the first evaluation, we compared the image quality with conventional HRCT among the FOVs $(40,80,160,240$, and $320 \mathrm{~mm})$ in the following items: bronchi $(n=66)$, small vessel ( $n=66)$, bronchiectasis $(n=6)$, bronchovascular bundle thickening $(n=4)$, consolidation $(n=4)$, emphysema $(n=5)$, faint nodules $(n=9)$, ground-glass opacity $(n=6)$, honeycombing $(n=9)$, 
Fig. 1 Images of conventional HRCT of cadaveric lung (usual interstitial pneumonia) at FOV $40 \mathrm{~mm}$ (a), $80 \mathrm{~mm}$ (b), $160 \mathrm{~mm}$ (c), $240 \mathrm{~mm}(\mathbf{d})$, and $320 \mathrm{~mm}(\mathbf{e})$. Sharpness of bronchi walls (arrowhead) and overall image quality were improved as the FOV was reduced from 320 to 240 or $160 \mathrm{~mm}$. However, when the FOV was reduced from 160 to 80 or $40 \mathrm{~mm}$, sharpness of bronchi walls (arrowhead) and overall image quality improved only slightly or were essentially unchanged
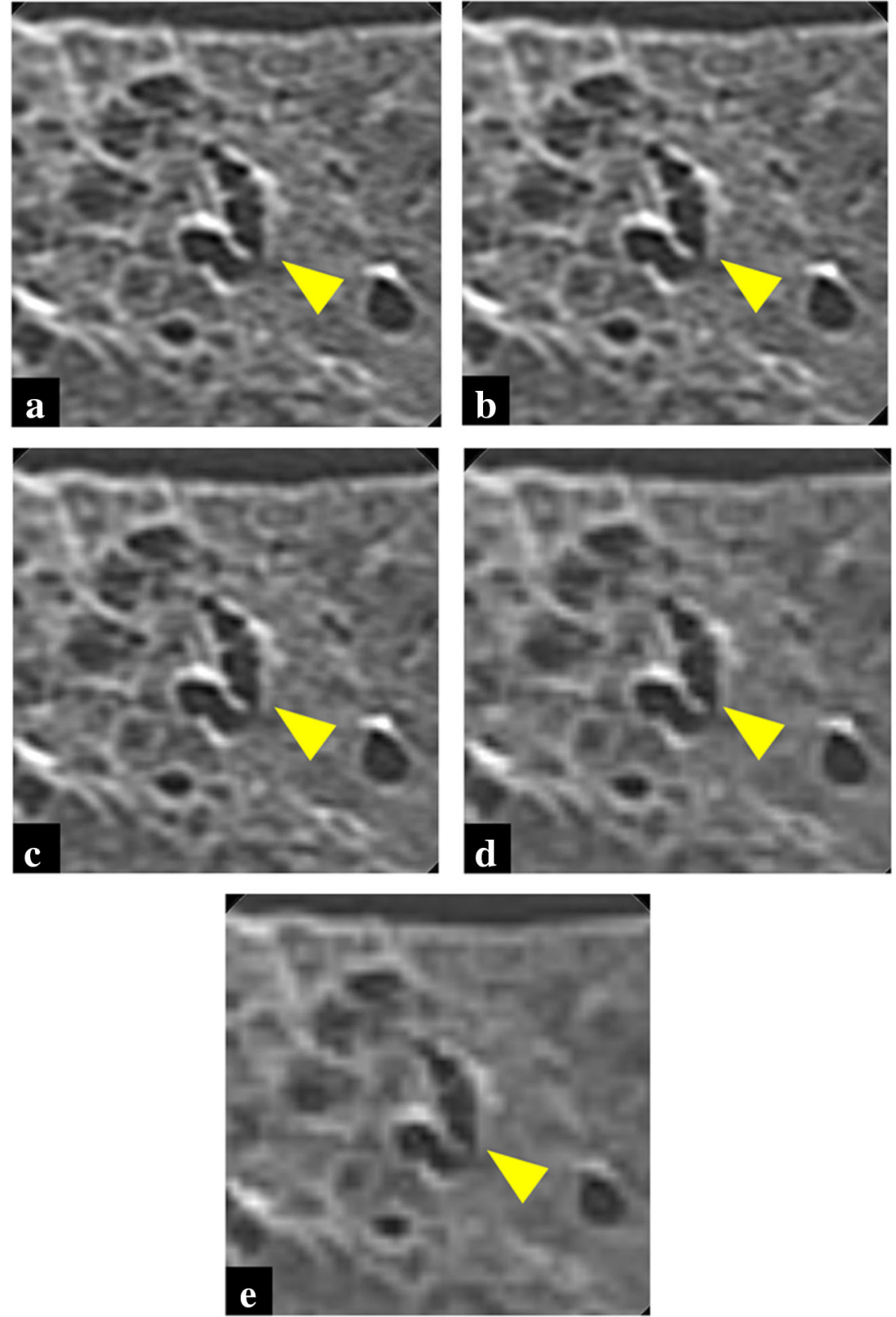

interlobular septal thickening $(n=7)$, reticulation $(n=6)$, and solid nodules $(n=14)$. These items were marked in advance to obtain uniform evaluation points. Overall image quality $(n=66)$ was evaluated on the whole image. An image was randomly selected from the five FOVs and evaluated with respect to the image with FOV $160 \mathrm{~mm}$ at the same level, independently by three radiologists (with 8,17 , and 25 years of experience) on a 5 point scale. The images were presented in random order and the evaluators were blinded to the next image to be presented.

The scoring scale was defined in comparison to the reference image as follows: score 1 , obviously poor image quality (almost impossible to detect structures or very difficult to clearly evaluate their margin or internal characteristics); score 2 , poor image quality (possible to detect structures but difficult to clearly evaluate their margin or internal characteristics); score 3, fair image quality (margin or internal characteristics can be detected and evaluated as well as in the reference image); score 4, better image quality (easier to detect structures and to evaluate their margin or internal characteristics); and score 5, excellent image quality (significantly easier to detect structures and to evaluate their margin or internal characteristics, without any indistinct findings).

\section{Evaluation of U-HRCT image quality}

In the second evaluation, the same comparisons were performed on U-HRCT images, using the same 5-point scale, by the same three radiologists in a blinded manner. 
Fig. 2 Images of U-HRCT of cadaveric lung (usual interstitial pneumonia) at FOV $40 \mathrm{~mm}$ (a), $80 \mathrm{~mm}$ (b), $160 \mathrm{~mm}(\mathbf{c}), 240 \mathrm{~mm}$ (d), and $320 \mathrm{~mm}$ (e). Sharpness of bronchi walls (arrowhead) and overall image quality were improved as the FOV was reduced from 320 to 240,160 , or $80 \mathrm{~mm}$. However, when the FOV was reduced from 80 to $40 \mathrm{~mm}$, sharpness of bronchi walls (arrowhead) and overall image improved only slightly
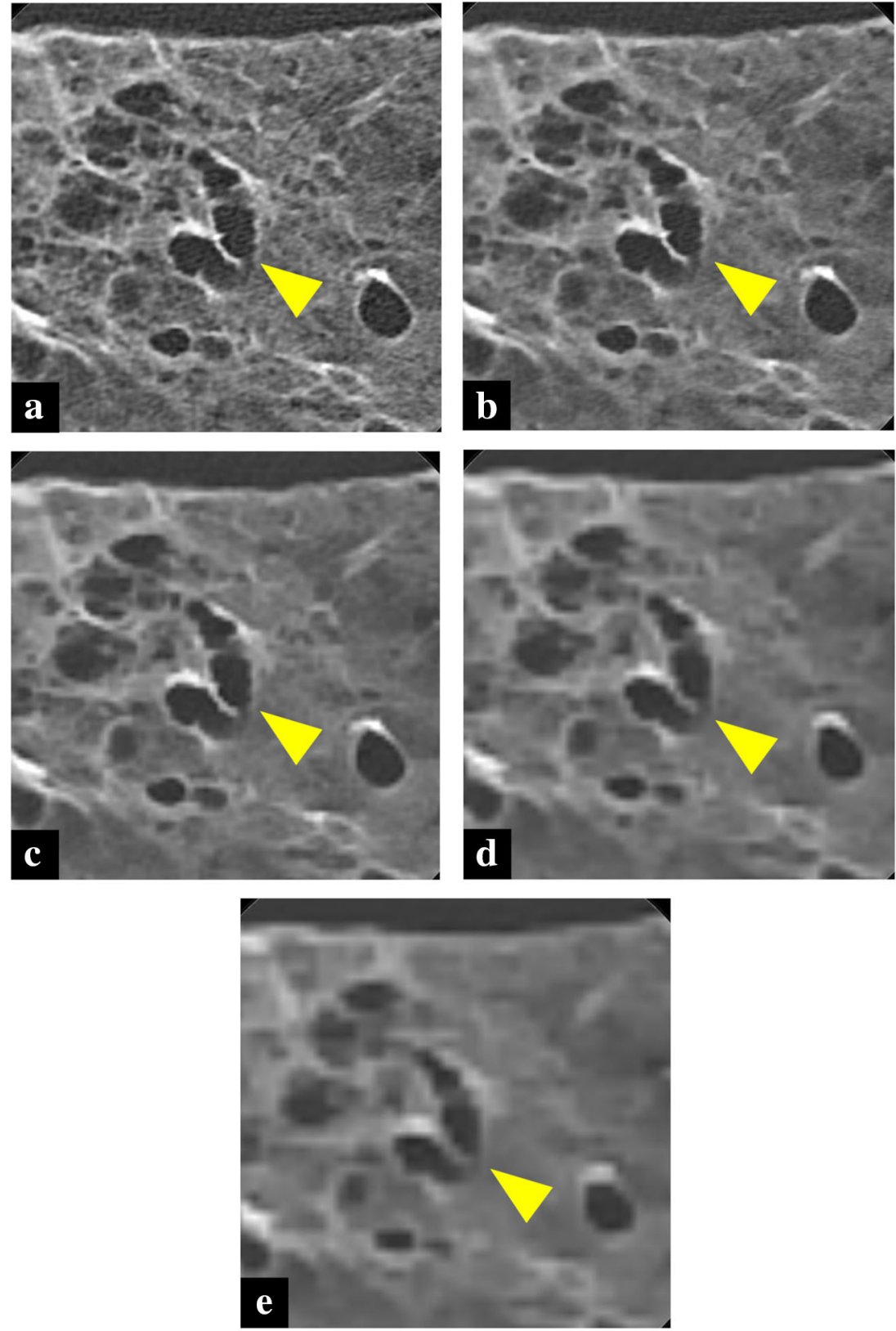

\section{Comparison of conventional HRCT and U-HRCT}

In the third evaluation, we selected one image obtained with conventional HRCT and the U-HRCT image of the same case, at the same FOV and slice level, and evaluated overall image quality. The two images were displayed side by side, with random assignment of the side and the order of image presentation. The image presented on the right side was evaluated with reference to the image presented on the left side. Overall image quality was evaluated on the whole image. The scoring was independently performed by three radiologists in comparison with the reference image using a 3-point scale: score 1, poor image quality (possible to detect structures but difficult to clearly evaluate their margin or internal characteristics); score 2, fair image quality (margin or internal characteristics can be detected and evaluated as well as in the reference image); and score 3 , excellent image quality (easier to detect structures and to evaluate their margin or internal characteristics, without any indistinct findings).

\section{Quantitative evaluation of noise}

Image noise was quantitatively obtained using the workstation by measuring the standard deviation (SD) values of circular regions of interest (ROI), which were drawn on the air portion of images. We used the same monitor as in the abovementioned evaluations. It has been reported that noise can be quantitatively evaluated by measuring the SD value with an $\mathrm{ROI}$ in the air 
portion [11-13]. Images for which ROIs could be drawn with all FOVs were extracted, and one to three ROIs of the same size were placed at the same location on the selected conventional HRCT image and the U-HRTC image. The SD value was calculated in cases with one ROI, and the average SD in cases with multiple ROIs.

\section{Statistical analysis}

In the first and second evaluation, the median values of the subjective scores of the three radiologists and the statistical significance of the differences among FOVs were analyzed using Shapiro-Wilk test to test normality and ANOVA with Bonferroni post hoc test. $P$ values $<0.005$ were considered significant. In the third evaluation, the median values of the subjective scores of the three radiologists and the statistical significance of the differences between conventional HRCT and U-HRCT at the same FOV were analyzed using Shapiro-Wilk test to test normality and two-way ANOVA with Bonferroni post hoc test. $P$ values $<0.05$ were considered significant. In the quantitative noise evaluation, two-way ANOVA and Bonferroni post hoc test were used to compare the various FOVs in each CT model ( $p<0.005$ considered significant) and the CT models at each FOV $(p<0.05$ considered significant). In all evaluations, the results of normality test were nonparametric. SPSS (version 24; IBM) was used for statistical processing.

\section{Results}

\section{Evaluation of conventional HRCT image quality}

The scores (FOV 40, 80, 160, 240, and $320 \mathrm{~mm}$ ) and the results of the comparisons between FOVs ( 40 vs. $80 \mathrm{~mm}, 40$ vs. $160 \mathrm{~mm}$, and 80 vs. $160 \mathrm{~mm}$ ) are summarized in Table 1, while the results of the other comparisons are summarized in Supplemental Table $1(F>27.2, p<0.001$ in all ANOVA comparisons).

Overall image quality scores clearly improved as the FOV was reduced from 320 to 240 or $160 \mathrm{~mm}$ (all $p<0.001$ ). However, when the FOV was reduced from 160 to $80 \mathrm{~mm}$, the score was not significantly different, and a further reduction from 80 to $40 \mathrm{~mm}$ led to a slight improvement $(p=0.002)$.

Likewise, the scores of all the evaluation items clearly improved as the FOV was reduced from 320 to $160 \mathrm{~mm}$ $(p<0.001)$. When the FOV was reduced from 160 to 80 or $40 \mathrm{~mm}$, the scores improved only slightly $(p<0.001)$ or showed no significant differences.

\section{Evaluation of U-HRCT image quality}

The scores for each FOV and the results of the comparison between the FOVs are summarized in Table 2, while the results of the other comparisons are shown in Supplemental Table 2 ( $F>80.8, p<0.001$ in all ANOVA comparisons).

The overall image quality scores clearly improved as the FOV was reduced from 320 to 240,160 , or $80 \mathrm{~mm}$ $(p<0.001)$. When the FOV was reduced from 80 to $40 \mathrm{~mm}$, the score improved slightly $(p<0.001)$.

Similarly, the scores of all the evaluations clearly improved as the FOV was reduced from 320 to $80 \mathrm{~mm}(p<0.001)$. However, when the FOV was further reduced from 80 to $40 \mathrm{~mm}$, the scores slightly improved $(p<0.001)$ or showed no significant differences.

\section{Comparison of conventional HRCT and U-HRCT}

The results are summarized in Table 3 (two-way ANOVA for the comparison among FOV sizes: $F=0.041, p=0.997$; twoway ANOVA analyzing CT model, FOV size, and their interaction: $F>5.6, p<0.001)$. The U-HRCT overall image quality scores were superior to those of conventional HRCT at all FOVs (all $p<0.001$, Figs. 3 and 4). A significant interaction between FOV size and CT model $(p<0.001)$ and a significant main effect of CT model $(p<0.001)$, but not of FOV ( $p=$ 0.997), were detected.

\section{Quantitative evaluation of noise}

The results are summarized in Table 4 (two-way ANOVA including FOV size, CT model, and their interaction: $F>235, p<0.001)$. In conventional HRCT, there were significant differences between all pairs of FOVs $(p<0.001)$, except between 40 and $80 \mathrm{~mm}(p=0.13)$. In U-HRCT, there were significant differences between all pairs of FOVs $(p<0.001)$. When comparing conventional HRCT with U-HRCT at each FOV, there were significant differences at FOV 40, 80, and $160 \mathrm{~mm}(p<0.001)$, but not at 240 and $320 \mathrm{~mm}(p=0.12$ and $p=0.13$, respectively). A significant interaction between FOV size and CT model was detected $(p<0.001)$, as well as significant main effects of both FOV size and CT model (both $p<0.001)$.

\section{Discussion}

Conventional HRCT image quality showed significant improvements when the FOV was reduced to $160 \mathrm{~mm}$, but further FOV reduction led to slight or nonsignificant improvements. Similar results were obtained for U-HRCT; however, significant improvements could be observed when the FOV was reduced to $80 \mathrm{~mm}$. Comparing U-HRCT and conventional 
Table 1 Subjective evaluation of conventional HRCT: CT findings
Score $($ mean $\pm \mathrm{SD})$

\begin{tabular}{|c|c|c|c|c|c|}
\hline \multirow[b]{2}{*}{ FOV size (mm) } & \\
\hline & 40 & 80 & 160 & 240 & 320 \\
\hline Bronchi & $3.5 \pm 0.5^{*, \#}$ & $3.3 \pm 0.4^{\dagger}$ & $3.0 \pm 0.0$ & $1.6 \pm 0.5$ & $1.0 \pm 0.0$ \\
\hline Small vessel & $3.4 \pm 0.5^{\#}$ & $3.2 \pm 0.4$ & $3.0 \pm 0.0$ & $1.7 \pm 0.4$ & $1.0 \pm 0.0$ \\
\hline Bronchiectasis & $3.3 \pm 0.5$ & $3.2 \pm 0.4$ & $3.0 \pm 0.0$ & $1.8 \pm 0.4$ & $1.0 \pm 0.0$ \\
\hline Bronchovascular bundle thickening & $3.3 \pm 0.4$ & $3.0 \pm 0.0$ & $3.0 \pm 0.0$ & $2.0 \pm 0.0$ & $1.0 \pm 0.0$ \\
\hline Consolidation & $3.8 \pm 0.4$ & $3.3 \pm 0.4$ & $3.0 \pm 0.0$ & $2.0 \pm 0.0$ & $1.3 \pm 0.4$ \\
\hline Emphysema & $3.0 \pm 0.0$ & $3.0 \pm 0.0$ & $3.0 \pm 0.0$ & $2.0 \pm 0.0$ & $1.0 \pm 0.0$ \\
\hline Faint nodule & $3.4 \pm 0.5$ & $3.1 \pm 0.3$ & $3.0 \pm 0.0$ & $1.9 \pm 0.3$ & $1.0 \pm 0.0$ \\
\hline Ground-glass opacity & $3.7 \pm 0.5$ & $3.2 \pm 0.4$ & $3.0 \pm 0.0$ & $1.8 \pm 0.4$ & $1.0 \pm 0.0$ \\
\hline Honeycombing & $3.2 \pm 0.3$ & $3.1 \pm 0.4$ & $3.0 \pm 0.0$ & $1.8 \pm 0.4$ & $1.0 \pm 0.0$ \\
\hline Interlobular septal thickening & $3.7 \pm 0.5^{\# \#}$ & $3.3 \pm 0.5$ & $3.0 \pm 0.0$ & $2.0 \pm 0.0$ & $1.0 \pm 0.0$ \\
\hline Reticulation & $3.8 \pm 0.4^{\#}$ & $3.7 \pm 0.5$ & $3.0 \pm 0.0$ & $2.0 \pm 0.0$ & $1.0 \pm 0.0$ \\
\hline Solid nodule & $3.3 \pm 0.5$ & $3.2 \pm 0.4$ & $3.0 \pm 0.0$ & $2.0 \pm 0.0$ & $1.0 \pm 0.0$ \\
\hline Overall image quality & $3.3 \pm 0.5^{* *, \#}$ & $3.1 \pm 0.3$ & $3.0 \pm 0.0$ & $1.9 \pm 0.3$ & $1.0 \pm 0.0$ \\
\hline
\end{tabular}

Data are presented as mean \pm SD. Subjective image analysis data were analyzed using Shapiro-Wilk test to test normality and ANOVA and Bonferroni post hoc tests. $p$ values $<0.005$ were considered significant. The scores of the evaluation items increased at a constant rate when the FOV was reduced to $160 \mathrm{~mm}$, but only slightly when the FOV was further reduced

* Significant difference between FOV $40 \mathrm{~mm}$ and FOV $80 \mathrm{~mm}(p=0.004) ;{ }^{* *}$ significant difference between FOV $40 \mathrm{~mm}$ and FOV $80 \mathrm{~mm}(p<0.001) ;{ }^{*}$ significant difference between FOV $40 \mathrm{~mm}$ and FOV $160 \mathrm{~mm}$ $(p<0.001) ;{ }^{\# \#}$ significant difference between FOV $40 \mathrm{~mm}$ and FOV $160 \mathrm{~mm}(p=0.002) ;{ }^{\dagger}$ significant difference between FOV $80 \mathrm{~mm}$ and FOV $160 \mathrm{~mm}(p=0.002)$

Table 2 Subjective evaluation of U-HRCT: CT findings

\begin{tabular}{|c|c|c|c|c|c|}
\hline \multirow[b]{2}{*}{ FOV size $(\mathrm{mm})$} & \multicolumn{5}{|c|}{ Score $($ mean $\pm \mathrm{SD})$} \\
\hline & 40 & 80 & 160 & 240 & 320 \\
\hline Bronchi & $4.6 \pm 0.5^{*, \#}$ & $4.0 \pm 0.0^{\dagger}$ & $3.0 \pm 0.0$ & $1.6 \pm 0.5$ & $1.0 \pm 0.0$ \\
\hline Small vessel & $4.5 \pm 0.5^{*, \#}$ & $4.1 \pm 0.3^{\dagger}$ & $3.0 \pm 0.0$ & $1.7 \pm 0.4$ & $1.0 \pm 0.0$ \\
\hline Bronchiectasis & $4.5 \pm 0.5^{\#}$ & $4.0 \pm 0.0^{\dagger}$ & $3.0 \pm 0.0$ & $1.8 \pm 0.4$ & $1.0 \pm 0.0$ \\
\hline Bronchovascular bundle thickening & $4.3 \pm 0.4^{\# \#}$ & $4.3 \pm 0.4^{\ddagger \ddagger}$ & $3.0 \pm 0.0$ & $2.0 \pm 0.0$ & $1.0 \pm 0.0$ \\
\hline Consolidation & $5.0 \pm 0.0^{\#}$ & $4.5 \pm 0.5^{\dagger}$ & $3.0 \pm 0.0$ & $2.0 \pm 0.0$ & $1.3 \pm 0.4$ \\
\hline Emphysema & $4.6 \pm 0.5^{\#}$ & $4.2 \pm 0.4^{\dagger}$ & $3.0 \pm 0.0$ & $2.0 \pm 0.0$ & $1.0 \pm 0.0$ \\
\hline Faint nodule & $4.1 \pm 0.6^{\#}$ & $4.1 \pm 0.3^{\dagger}$ & $3.0 \pm 0.0$ & $1.9 \pm 0.3$ & $1.0 \pm 0.0$ \\
\hline Ground-glass opacity & $4.7 \pm 0.5^{\#}$ & $4.2 \pm 0.4^{\dagger}$ & $3.0 \pm 0.0$ & $1.8 \pm 0.4$ & $1.0 \pm 0.0$ \\
\hline Honeycombing & $4.3 \pm 0.5^{\#}$ & $4.0 \pm 0.0^{\dagger}$ & $3.0 \pm 0.0$ & $1.8 \pm 0.4$ & $1.0 \pm 0.0$ \\
\hline Interlobular septal thickening & $4.6 \pm 0.5^{* *, \#}$ & $4.0 \pm 0.0^{\dagger}$ & $3.0 \pm 0.0$ & $2.0 \pm 0.0$ & $1.0 \pm 0.0$ \\
\hline Reticulation & $4.7 \pm 0.5^{\#}$ & $4.2 \pm 0.4^{\dagger}$ & $3.0 \pm 0.0$ & $2.0 \pm 0.0$ & $1.0 \pm 0.0$ \\
\hline Solid nodule & $4.3 \pm 0.5^{\#}$ & $4.0 \pm 0.0^{\dagger}$ & $3.0 \pm 0.0$ & $2.0 \pm 0.0$ & $1.0 \pm 0.0$ \\
\hline Overall image quality & $4.5 \pm 0.5^{*, \#}$ & $4.0 \pm 0.2^{\dagger}$ & $3.0 \pm 0.0$ & $1.9 \pm 0.3$ & $1.0 \pm 0.0$ \\
\hline
\end{tabular}

Data are presented as mean \pm SD. Subjective image analysis data were analyzed using Shapiro-Wilk test to test normality and ANOVA and Bonferroni post hoc tests. $p$ values $<0.005$ were considered significant. The scores of the evaluation items increased at a constant rate when the FOV was reduced to $80 \mathrm{~mm}$, but only slightly when the FOV was further reduced

* Significant difference between FOV $40 \mathrm{~mm}$ and FOV $80 \mathrm{~mm}(p<0.001) ;{ }^{* *}$ significant difference between FOV $40 \mathrm{~mm}$ and FOV $80 \mathrm{~mm}(p=0.001) ;{ }^{\#}$ significant differences between FOV $40 \mathrm{~mm}$ and FOV $160 \mathrm{~mm}$ $(p<0.001) ;{ }^{\# \#}$ significant difference between FOV $40 \mathrm{~mm}$ and FOV $160 \mathrm{~mm}(p=0.001) ;{ }^{\dagger}$ significant difference between FOV $80 \mathrm{~mm}$ and FOV $160 \mathrm{~mm}(p<0.001) ;{ }^{\dagger \uparrow}$ significant difference between FOV $80 \mathrm{~mm}$ and FOV $160 \mathrm{~mm}(p=0.001)$ 
Table 3 Subjective comparison of U-HRCT and conventional HRCT: CT findings

Score $($ mean $\pm \mathrm{SD})$

\begin{tabular}{|c|c|c|c|c|c|c|}
\hline FOV size $(\mathrm{mm})$ & & 40 & 80 & 160 & 240 & 320 \\
\hline \multirow[t]{2}{*}{ Overall image quality } & U-HRCT & $3.0 \pm 0.0^{*}$ & $3.0 \pm 0.0^{*}$ & $3.0 \pm 0.0 *$ & $3.0 \pm 0.0^{*}$ & $2.9 \pm 0.4^{*}$ \\
\hline & C-HRCT & $1.0 \pm 0.0$ & $1.0 \pm 0.0$ & $1.0 \pm 0.0$ & $1.0 \pm 0.0$ & $1.1 \pm 0.4$ \\
\hline
\end{tabular}

Data are presented as mean $\pm \mathrm{SD}$. The scores of the subjective overall image quality analysis were analyzed using Shapiro-Wilk test to test normality and twoway ANOVA and Bonferroni post hoc tests comparing conventional HRCT and U-HRCT at the same FOV. $P$ values $<0.005$ were considered significant

*Significant difference between U-HRCT and conventional HRCT (C-HRCT) $(p<0.001)$

HRCT, the U-HRCT overall image quality scores were superior to those of conventional HRCT at all FOVs. The image noise of U-HRCT, but not conventional HRCT, was increased when the FOV was reduced from 80 to $40 \mathrm{~mm}$.

The spatial resolution of conventional HRCT is in the range of $0.23-0.35 \mathrm{~mm}[5,9,10]$. When using a $512 \times 512$ matrix size, the size of one pixel at FOV $160 \mathrm{~mm}(80 \mathrm{~mm})$ is $0.313 \mathrm{~mm}(0.156 \mathrm{~mm})$. Therefore, the spatial resolution of conventional HRCT is higher than the pixel size at FOV $160 \mathrm{~mm}$ and lower at FOV $80 \mathrm{~mm}$. These considerations are relevant to the quantitative evaluation of noise. It has been reported that image quality is improved and the noise is enhanced at higher resolution [3, 4]. We can conclude that in conventional HRCT improvements in image quality cannot be expected, and noise would not significantly increase, when the FOV is reduced from 80 to $40 \mathrm{~mm}$, because the pixel size becomes smaller than the resolution. In U-HRCT, the spatial resolution is $0.14 \mathrm{~mm}[5,7-9]$. When using a $512 \times 512 \mathrm{ma}-$ trix size, the pixel size at FOV $40 \mathrm{~mm}$ is $0.078 \mathrm{~mm}$. Therefore, the spatial resolution of U-HRCT is higher (lower) than the pixel size at FOV $80 \mathrm{~mm}(40 \mathrm{~mm})$. Therefore, improvements in U-HRCT image quality can be expected as the FOV is reduced to $80 \mathrm{~mm}$, because the resolution of U-HRCT is still higher than the pixel size, and the same applies to image noise. The quantitative evaluation of noise showed a synergic effect of FOV size and CT model, and both factors affected image noise independently. In particular, U-HRCT noise becomes more severe compared with conventional HRCT when the FOV is reduced. At FOV 240 and $320 \mathrm{~mm}$, noise is almost
Fig. 3 Images of conventional HRCT $(\mathbf{a}, \mathbf{c})$ and U-HRCT $(\mathbf{b}, \mathbf{d})$ of cadaveric lung (usual interstitial pneumonia) at FOV $40 \mathrm{~mm}$. Overall image quality of UHRCT was improved compared with conventional HRCT and noise was more severe in UHRCT compared with conventional HRCT
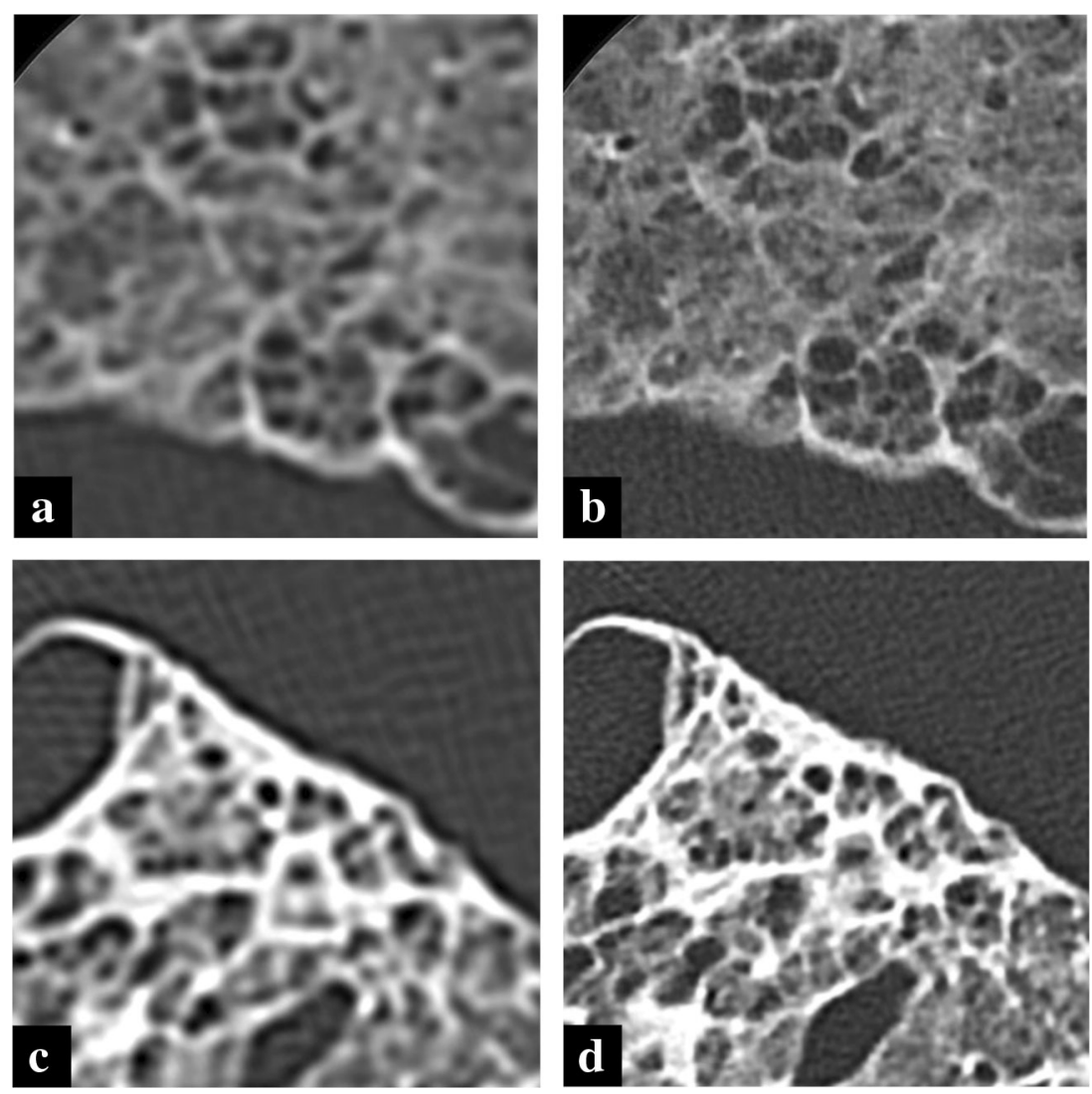
Fig. 4 Images of conventional HRCT $(\mathbf{a}, \mathbf{c})$ and U-HRCT $(\mathbf{b}, \mathbf{d})$ of cadaveric lung (usual interstitial pneumonia) at FOV $320 \mathrm{~mm}$. Overall image quality was improved in U-HRCT compared with conventional HRCT and no difference in noise was detected
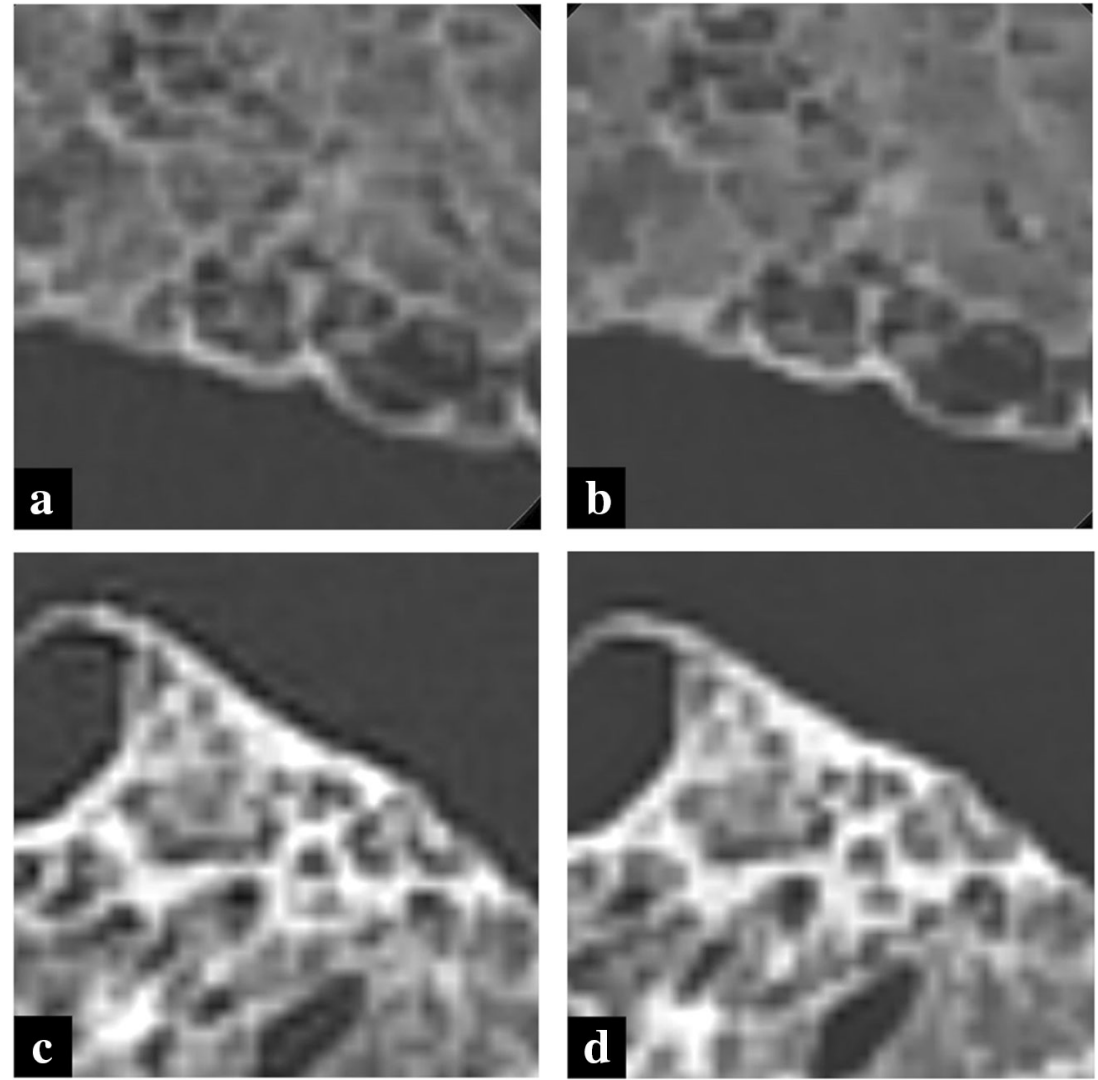

equal in U-HRCT and HRCT, suggesting that the noise increase associated with U-HRCT might not affect clinical practice, where these or larger FOVs are frequently used.

Therefore, the use of U-HRCT allows clearer diagnostic images compared with conventional HRCT, without increasing noise. A study reported that in U-HRCT, minute structures such as bronchi were clearly delineated, and noise was reduced, in comparison with conventional HRCT [14]. It was also reported that images acquired using U-HRCT with a 0.25 mm slice thickness showed improved performance compared with a $0.5-\mathrm{mm}$ thickness in visualizing the Adamkiewicz artery [15] and allowed visualizing calcified lesions of the coronary artery with fewer artifacts [16]. These reports support the results of our study. Regarding structures such as nodules, the average interslice volume was reported to be reduced, and small nodule detection improved, by using thin sections [17].

Image quality affects the quantitative and qualitative evaluation of lung field lesions [18, 19]. Improvement in image quality in the lung field plays an important role in diagnosis. Studies reported that U-HRCT can improve nodule images in the lung or bronchi, thereby improving diagnostic confidence $[6,20]$. It was also reported that when using U-HRCT with a small FOV $(100 \mathrm{~mm})$, the lung nodule image quality and diagnostic confidence were improved compared with conventional HRCT with a larger FOV (350 mm) [18]. In summary, U-HRCT can be expected to improve the quality of diagnostic imaging of the lung and reduce the noise in clinical practice by allowing reduced FOVs.

Table 4 Quantitative evaluation of noise on conventional HRCT and U-HRCT: SD value

\begin{tabular}{|c|c|c|c|c|c|c|}
\hline \multirow[b]{2}{*}{ FOV size $(\mathrm{mm})$} & \multicolumn{6}{|c|}{ Score $($ mean $\pm \mathrm{SD})$} \\
\hline & & 40 & 80 & 160 & 240 & 320 \\
\hline \multirow[t]{2}{*}{ Noise } & U-HRCT & $42.0 \pm 6.4^{*}$ & $29.7 \pm 4.2^{*}$ & $17.8 \pm 1.7^{*}$ & $9.4 \pm 0.9$ & $5.9 \pm 0.6$ \\
\hline & C-HRCT & $20.4 \pm 6.5$ & $19.2 \pm 6.9$ & $15.3 \pm 4.8$ & $10.2 \pm 3.1$ & $6.7 \pm 1.7$ \\
\hline
\end{tabular}

The SD values are presented as mean $\pm \mathrm{SD}$. Quantitative noise evaluation data were analyzed using two-way ANOVA and Bonferroni post hoc tests. $p$ values $<0.005$ were considered significant. In U-HRCT, there were significant differences among all FOVs $(p<0.001)$. In conventional HRCT, all differences were significant except that between FOV 40 and $80 \mathrm{~mm}(p=0.13)$

*At FOV 40, 80, and $160 \mathrm{~mm}$, there were significant differences between conventional HRCT and U-HRCT $(p<0.001)$ 
This study has some limitations. Cadaveric lungs were used, and we did not evaluate the effects of absorption and scattering in the thorax on image quality. Moreover, image evaluation using cadaveric lungs does not include respiratory variation or fluctuations of the chest wall. Thus, image acquisition in this study does not necessarily correspond to actual clinical practice, making unclear to what extent our results can be applied to clinical practice; it will be necessary to study the influence of these fluctuations on U-HRCT image quality. Moreover, the sample size was relatively small. Regarding the evaluation procedure, the radiologists who evaluated the various items may have been unconsciously influenced by other information such as noise. Thus, it is possible that the evaluations of the different items were not completely independent. The side-by-side comparison used to evaluate conventional HRCT and U-HRCT may bias the radiologist's evaluation. Only Canon CT scanners were used in the study, and therefore, strictly speaking, the results can be applied to UHRCT developed by Canon Medical Systems, and it is unknown whether the same results would apply to U-HRCT developed by other companies. Despite our best efforts to match the slice position, the match was not perfect, so that the slice positions of the evaluation image and the reference image were slightly different, and such slight difference in slice position may have affected the image quality evaluation. It was reported that the FC81 reconstruction kernel performs different processes between conventional HRCT and U-HRCT [14], and such difference in reconstruction may affect image quality. We used pathologically diagnosed cadaveric lungs to our study, but we did not correlate abnormal CT findings such as solid nodules with the pathological assessments. The cadaveric lungs were inflated and fixed using the Heitzman's method, and we do not know in detail the effects of such procedure on lung tissue, which could in principle affect both pathological diagnosis and image evaluation. However, the conventional HRCT and U-HRCT images were acquired almost at the same time, so that there was no difference in the quality of the cadaveric lungs between conventional HRCT and UHRCT. A method to evaluate image quality by measuring tumor SNR has been reported [21], but SNR was not measured in this study. Because some evaluation items were not uniform (such as honeycombing, reticulation, etc.), unlike in tumors, when setting ROIs, the signal varied with the evaluation items, leading to instability of the quantitative evaluation.

In conclusion, since the spatial resolution of U-HRCT is higher than that of conventional HRCT, the limit of FOV at which image quality improves with conventional HRCT is $160 \mathrm{~mm}$, while with U-HRCT image quality improves even when the FOV is reduced to $80 \mathrm{~mm}$. Moreover, at all FOVs, U-HRCT image quality is improved compared with conventional HRCT. Thus, improvement of clinical diagnostic performance may be expected using U-HRCT, although further clinical studies will be needed.
Funding information Noriyuki Tomiyama received a research grant from Canon Medical Systems Corp. The other authors state that they have not received any funding for this work.

\section{Compliance with ethical standards}

Guarantor The scientific guarantor of this publication is Noriyuki Tomiyama.

Conflict of interest Noriyuki Tomiyama received a research grant from Canon Medical Systems Corp., formerly Toshiba Medical Systems. This work was technically supported by Canon Medical Systems Corp. Tomo Miyata, Masahiro Yanagawa, Akinori Hata, Osamu Honda, Noriko Kikuchi, Yuriko Yoshida, and Mitsuko Tsubamoto have no conflict of interest related to this study.

Statistics and biometry No complex statistical methods were necessary for this paper.

Informed consent Written informed consent was waived by the Institutional Review Board.

Ethical approval Institutional Review Board approval was obtained.

Study subjects or cohorts overlap The same study subjects or cohorts were previously reported in European Radiology 2018; 28:5060-5068 because cadaveric lungs stored in our institution were used in this study.

\section{Methodology \\ - Prospective \\ - Experimental \\ - Performed at one institution}

Open Access This article is licensed under a Creative Commons Attribution 4.0 International License, which permits use, sharing, adaptation, distribution and reproduction in any medium or format, as long as you give appropriate credit to the original author(s) and the source, provide a link to the Creative Commons licence, and indicate if changes were made. The images or other third party material in this article are included in the article's Creative Commons licence, unless indicated otherwise in a credit line to the material. If material is not included in the article's Creative Commons licence and your intended use is not permitted by statutory regulation or exceeds the permitted use, you will need to obtain permission directly from the copyright holder. To view a copy of this licence, visit http://creativecommons.org/licenses/by/4.0/.

\section{References}

1. Seemann MD, Staebler A, Beinert T et al (1999) Usefulness of morphological characteristics for the differentiation of benign from malignant solitary pulmonary lesions using HRCT. Eur Radiol 9: 409-417

2. Zwirewich CV, Vedal S, Miller RR, Muller NL (1991) Solitary pulmonary nodule: high-resolution $\mathrm{CT}$ and radiologic-pathologic correlation. Radiology 179:469-476

3. Barrett JF, Keat N (2004) Artifacts in CT: recognition and avoidance. Radiographics 6:1679-1691

4. Boas FE, Fleischmann D (2012) CT artifacts: causes and reduction techniques. Imaging Med 4:229-240 
5. Kakinuma R, Moriyama N, Muramatsu Y et al (2015) Ultra-highresolution computed tomography of the lung: image quality of a prototype scanner. PLoS One 10:e0137165

6. Zhu H, Zhang L, Wang Y et al (2017) Improved image quality and diagnostic potential using ultra-high-resolution computed tomography of the lung with small scan FOV: a prospective study. PLoS One 12:e0172688

7. Hata A, Yanagawa M, Honda O et al (2018) Effect of matrix size on the image quality of ultra-high-resolution CT of the lung: comparison of $512 \times 512,1024 \times 1024$, and $2048 \times 2048$. Acad Radiol 25: 869-876

8. Yanagawa M, Tomiyama N, Honda O et al (2010) Multidetector CT of the lung: image quality with garnet-based detectors. Radiology 255:944-954

9. Tsukagoshi S, Ota T, Fujii M, Kazama M, Okumura M, Johkoh T (2007) Improvement of spatial resolution in the longitudinal direction for isotropic imaging in helical CT. Phys Med Biol 52:791-801

10. Markarian B, Dailey ET (1993) Preparation of inflated lung specimens. In: Groskin SA (ed) Heitzman's the lung: radiologicpathologic correlations. Mosby, St. Louis, pp 4-12

11. Boehm T, Willmann JK, Hilfiker PR et al (2003) Thin-section CT of the lung: dose electrocardiographic triggering influence diagnosis? Radiology 229:483-491

12. Baumueller S, Winklehner A, Karlo C et al (2012) Low-dose CT of the lung: potential value of iterative reconstructions. Eur Radiol 22: 2597-2606

13. Booij R, Dijkshoorn ML, van Straten M (2017) Efficacy of a dynamic collimator for overranging dose reduction in a second- and third-generation dual source CT scanner. Eur Radiol 27:3618-3624

14. Yanagawa M, Hata A, Honda O et al (2018) Subjective and objective comparisons of image quality between ultra-high-resolution
$\mathrm{CT}$ and conventional area detector $\mathrm{CT}$ in phantoms and cadaveric human lungs. Eur Radiol 28:5060-5068

15. Yoshioka K, Tanaka R, Takagi H et al (2018) Ultra-high-resolution CT angiography of the artery of Adamkiewicz: a feasibility study. Neuroradiology 60:109-115

16. Motoyama S, Ito H, Sarai M et al (2018) Ultra-high-resolution computed tomography angiography for assessment of coronary artery stenosis. Circ J 82:1844-1851

17. Fischbach F, Knollmann F, Griesshaber V, Freund T, Akkol E, Felix R (2003) Detection of pulmonary nodules by multislice computed tomography: improved detection rate with reduced slice thickness. Eur Radiol 13:2378-2383

18. Goo JM, Tongdee T, Tongdee R, Yeo K, Hildebolt CF, Bae KT (2005) Volumetric measurement of synthetic lung nodules with multi-detector row CT: effect of various image reconstruction parameters and segmentation thresholds on measurement accuracy. Radiology 235:850-856

19. Zerhouni EA, Spivey JF, Morgan RH, Leo FP, Stitik FP, Siegelman SS (1982) Factors influencing quantitative CT measurements of solitary pulmonary nodules. J Comput Assist Tomogr 6:1075-1087

20. Sheshadri A, Rodriguez A, Chen R et al (2015) Effect of reducing field of view on multidetector quantitative computed tomography parameters of airway wall thickness in asthma. J Comput Assist Tomogr 39:584-590

21. Beer L, Toepker M, Ba-Ssalamah A et al (2019) Objective and subjective comparison of virtual monoenergetic vs. polychromatic images in patients with pancreatic ductal adenocarcinoma. Eur Radiol 29:3617-3625

Publisher's note Springer Nature remains neutral with regard to jurisdictional claims in published maps and institutional affiliations. 